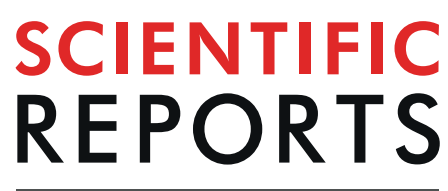

natureresearch

\title{
eGFR and deep white matter hyperintensity as predictors of cognitive decline long after carotid endarterectomy
}

\author{
Akira Nakamizo $^{1 *}$, Toshiyuki Amano ${ }^{1}$, Takahiro Kuwashiro² ${ }^{2}$ Masahiro Yasaka ${ }^{2} \&$ \\ Yasushi Okada ${ }^{2}$
}

Chronic kidney disease and white matter hyperintensity (WMH) are associated with cognitive decline. The aim of this study was to assess the correlations between estimated glomerular filtration rate (eGFR) or WMH and cognitive function in patients who have undergone carotid endarterectomy (CEA). Cognitive functions were investigated using the Neurobehavioral Cognitive Status Examination (Cognistat) in 83 patients who had undergone CEA. The eGFR at 5 years prior to examination was significantly associated with severe cognitive impairment (odds ratio, 0.89 per $1-\mathrm{mL} / \mathrm{min} / 1.73 \mathrm{~m}^{2}$ increase, $95 \%$ confidence interval $0.82-0.97, p=0.0004$ ). Receiver operating characteristic analysis revealed that a cutoff eGFR of $46.8 \mathrm{~mL} / \mathrm{min} / 1.73 \mathrm{~m}^{2}$ at 5 years prior to examination offered the most reliable predictor of severe cognitive impairment (sensitivity $88.9 \%$, specificity $76.5 \%$, area under the curve 0.848 ). The eGFR at 5 years prior to examination showed a significant linear association with total Cognistat score $\left(r^{2}=0.11035, p=0.0032\right)$ compared to eGFR at 3 years prior to examination $\left(r^{2}=0.06455, p=0.0230\right)$ or at examination $\left(r^{2}=0.0210, p=0.0210\right)$. Spearman's correlation coefficient revealed that orientation, comprehension, repetition, construction, memory, and similarity correlated with eGFR at 5 years prior to examination. Conversely, Fazekas grade for deep WMH at examination was associated with total Cognistat score $(p=0.0016)$, unlike that at 3 years $(p=0.0100)$ or 5 years prior to examination $(p=0.0172)$. While eGFR correlates with future cognitive function, deep WMH associates with present cognitive function in patients who have undergone CEA.

Carotid stenosis shares various risk factors common for cognitive decline such as hypertension, diabetes, and dyslipidemia, but cognitive function is rarely considered in evaluating or treating carotid stenosis. Carotid endarterectomy (CEA) might theoretically improve or maintain cognitive function by restoring normal cerebral perfusions and by removing the source of microemboli immediately after surgery. However, CEA reduced microemboli only up to $70 \%$ at 1 year after surgery in 28 patients ${ }^{1}$, suggesting that risk of cognitive decline persists even after CEA. Moreover, microemboli during surgical maneuvers, hypoperfusion during carotid artery clamping, general anesthesia, and postoperative hyperperfusion can cause postoperative cognitive declines ${ }^{2}$. Taken together, CEA can result in improvement or deterioration of cognitive function, and whether these complex interactions ultimately result in cognitive improvement or decline remains unclear ${ }^{3}$. In the long term, various cerebrovascular risk factors might influence cognitive function after CEA. Periodic cognitive assessment thus appears necessary to detect early cognitive changes long after CEA.

Several studies have reported that age, education, intelligence quotient, brain atrophy, preoperative symptoms, and white matter hyperintensity (WMH) are associated with cognitive changes after CEA, but most such studies were performed in the early postoperative stage, mainly at only $1-6$ months after CEA ${ }^{4-6}$. CEA prevented expected increases in WMH at 1 year after CEA in 14 patients with preoperative cognitive decline ${ }^{1}$, but the effects of the existing WMH on long-term cognitive change are uncertain. Chronic kidney disease (CKD) is also known to increase the risk of cognitive decline $\mathrm{e}^{7,8}$. Low estimated glomerular filtration rate (eGFR) was associated

${ }^{1}$ Department of Neurosurgery, Clinical Research Institute, National Hospital Organization Kyushu Medical Center, Fukuoka, Japan. ${ }^{2}$ Department of Cerebrovascular Medicine and Neurology, Clinical Research Institute, National Hospital Organization Kyushu Medical Center, Fukuoka, Japan. *email: nakamizo.akira.gv@mail.hosp.go.jp 


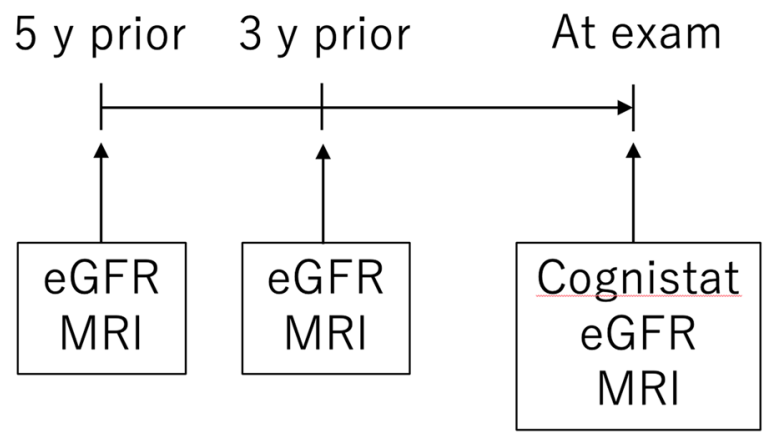

Figure 1. Flowchart of the study.

with dysexecutive and amnestic mild cognitive impairment in 622 subjects who were $\geq 70$ years old ${ }^{9}$, but little is known about the association between eGFR and cognitive function long after CEA.

The aim of this study was to assess the associations between eGFR or WMH and cognitive function in patients who have undergone CEA, particularly in terms of whether eGFR and WMH are useful to predict cognitive declines occurring within several years.

\section{Material and Methods}

Patients. Eighty-three patients who had undergone CEA and were admitted to our institution from February 2016 to October 2018 for periodic follow-up were invited to participate in this study. This study was approved by the National Hospital Organization Kyushu Medical Center Ethical Review Board and performed in accordance with the relevant guidelines and regulations, and all patients provided written informed consent prior to enrolment. This study represented a retrograde analysis of prospectively collected data. Figure 1 shows a flowchart for participation in this study. All patients underwent CEA for severe carotid stenosis or unstable plaque under general anesthesia with propofol. Surgical indications for carotid stenosis were as follows: linear stenosis $>70 \%$, area stenosis $>90 \%$, and peak systolic flow velocity of cervical internal carotid artery (ICA) $>200 \mathrm{~cm} / \mathrm{s}$ on colorcoded sonography. Twenty-three patients with carotid stenosis $>50 \%$ who were treated conservatively were also investigated as a reference group.

Cognitive assessment. The Japanese versions of Cognistat ${ }^{10}$ was adopted to assess cognitive functions. Cognistat consists of 10 sub-components: orientation, attention, comprehension, repetition, naming, construction, memory, calculation, similarities, and judgment ${ }^{11}$. Severity of impairment in each cognitive domain is defined as follows: score $\geq 9$, normal; 8 , mild impairment; 7 , moderate impairment; and $\leq 6$, severe impairment ${ }^{10}$. Patients who had undergone Cognistat previously were not included in this study, to avoid any practice effect.

Magnetic resonance imaging (MRI). All patients underwent 1.5-T MRI to assess brain WMH. Deep $\mathrm{WMH}$ and periventricular hyperintensity $(\mathrm{PVH})$ on T2-weighted imaging were classified using Fazekas grade ${ }^{12}$. Intracranial atherosclerosis was defined as $>50 \%$ stenosis of the ICA, middle cerebral artery (MCA), or anterior cerebral artery (ACA) on magnetic resonance angiography (MRA).

Ultrasound. All patients underwent cervical ultrasound to measure carotid stenosis using the European Carotid Surgery Trial method. Postoperative hyperperfusion state was defined as a $>1.5$-fold increase in mean flow velocity of the MCA compared to the preoperative value on transcranial color-coded sonography.

Statistical analysis. Except where indicated otherwise, all results are reported as mean \pm standard deviation. Statistical analysis was performed using the Wilcoxon rank-sum test for non-parametric data. Differences in binary variables were assessed using Pearson's chi-square test. Logistic regression models were used to evaluate independent associations between variables and cognitive decline. Cutoff values were defined for eGFR. Sensitivity and specificity were calculated to predict severe impairment on Cognistat. Linear regression analysis was performed for paired eGFR and Cognistat scores. The correlation between Cognistat scores and eGFR was assessed using Spearman's correlation coefficient. Kruskal-Wallis test was used to assess differences in Cognistat scores between Fazekas grades for deep WMH. Differences were considered significant for values of $\mathrm{p}<0.05$. All statistical analyses were performed using JMP Pro version 13.2 software (SAS Institute, Cary, NC).

\section{Results}

Patients. Participants comprised 83 patients with a history of CEA (68 men, 15 women; mean age at examination, $76.5 \pm 5.9$ years; mean follow-up between CEA and examination, $6.7 \pm 3.1$ years). Preoperative presentation was symptomatic in 50 patients $(60.2 \%)$, comprising brain infarction in 30 , transient ischemic attack in 11 , and monocular blindness in 8 , and seizure in 1 . Among symptomatic patients, mean interval from onset to CEA was 2.2 months. No significant differences in the number of severely impaired cognitive domains on Cognistat or total Cognistat score were seen between symptomatic and asymptomatic patients $(p=0.5489$ and $p=0.6647$, respectively). During follow-up, two patients suffered ipsilateral lacunar infarction, at 5 and 7 years after CEA, but no patients experienced recurrent ipsilateral symptoms due to restenosis. Eighteen patients (21.7\%) showed brain infarction $>3 \mathrm{~cm}$ in maximum diameter at cognitive examination. No significant differences in the number 


\begin{tabular}{|c|c|c|c|c|c|c|}
\hline & \multirow{2}{*}{$\begin{array}{l}\text { Overall } \\
(\mathbf{n}=\mathbf{8 3})\end{array}$} & \multicolumn{5}{|c|}{ Number of severely impaired cognitive domains on Cognistat } \\
\hline & & None $(\mathbf{n}=36)$ & $1(n=28)$ & $2(\mathrm{n}=9)$ & $\geq 3(n=10)$ & P value \\
\hline Age at examination (years) & $76.5 \pm 5.9$ & $75.9 \pm 5.8$ & $74.1 \pm 5.5$ & $81.1 \pm 3.9$ & $81.2 \pm 3.5$ & 0.0002 \\
\hline Education (years) & $12.9 \pm 2.9$ & $14.2 \pm 2.1$ & $12.5 \pm 2.8$ & $10.8 \pm 3.4$ & $11.6 \pm 3.3$ & 0.0056 \\
\hline Follow-up period (years) & $6.7 \pm 3.1$ & $6.3 \pm 2.8$ & $6.8 \pm 3.2$ & $6.4 \pm 3.0$ & $8.2 \pm 3.9$ & 0.5533 \\
\hline Male (\%) & $68(81.9)$ & $29(80.6)$ & $22(78.6)$ & $9(100)$ & $8(80.0)$ & 0.5184 \\
\hline Symptomatic presentation (\%) & $50(60.2)$ & $20(55.6)$ & $18(64.3)$ & $7(77.8)$ & $5(50.0)$ & 0.5489 \\
\hline \multicolumn{7}{|l|}{ Medical history (\%) } \\
\hline Hypertension & 78 (94.0) & $36(100)$ & $25(89.3)$ & $7(77.8)$ & $10(100)$ & 0.0419 \\
\hline Diabetes mellitus & $40(48.2)$ & $17(47.2)$ & $14(50.0)$ & $5(55.6)$ & $4(40.0)$ & 0.9157 \\
\hline Dyslipidemia & 76 (91.6) & $33(91.7)$ & $25(89.3)$ & $8(88.9)$ & $10(100)$ & 0.7545 \\
\hline Atherosclerosis obliterans & $8(9.6)$ & $2(5.6)$ & $3(10.7)$ & 0 & $3(30.0)$ & 0.0918 \\
\hline Ischemic heart disease & $38(45.8)$ & $13(36.1)$ & $13(46.4)$ & $5(55.6)$ & $7(70.0)$ & 0.2540 \\
\hline Atrial fibrillation & $4(4.8)$ & $1(2.8)$ & $3(10.7)$ & 0 & 0 & 0.3326 \\
\hline Smoking history & $63(75.9)$ & $28(77.8)$ & $18(64.3)$ & $8(88.9)$ & $9(90.0)$ & 0.2559 \\
\hline Current smoking & $10(12.1)$ & $4(11.1)$ & $4(14.3)$ & $1(11.1)$ & $1(10.0)$ & 0.9761 \\
\hline Alcohol $>40 \mathrm{~g} /$ day & $5(6.0)$ & 0 & $4(14.3)$ & 0 & $1(10.0)$ & 0.0881 \\
\hline Use of antiplatelet & 81 (97.6) & $35(97.2)$ & $27(96.4)$ & $9(100)$ & $10(100)$ & 0.8848 \\
\hline Use of anticoagulant & $6(7.2)$ & $2(5.6)$ & $4(14.3)$ & 0 & 0 & 0.2945 \\
\hline Dementia & $3(3.6)$ & 0 & $2(7.1)$ & 0 & $1(10.0)$ & 0.2772 \\
\hline Intracranial atherosclerosis & $18(21.7)$ & $6(16.7)$ & $4(14.3)$ & $4(44.4)$ & $4(40.0)$ & 0.1042 \\
\hline \multicolumn{7}{|l|}{ CA stenosis at examination (\%) } \\
\hline Right & $37.4 \pm 26.3$ & $35.5 \pm 25.2$ & $34.9 \pm 30.2$ & $51.7 \pm 10.3$ & $38.4 \pm 27.7$ & 0.4247 \\
\hline Left & $37.4 \pm 27.3$ & $36.5 \pm 29.2$ & $45.7 \pm 22.6$ & $21.3 \pm 25.9$ & $32.2 \pm 29.1$ & 0.1811 \\
\hline $\mathrm{mRS}$ at examination & $0.5 \pm 0.9$ & $0.4 \pm 0.8$ & $0.4 \pm 0.8$ & $0.4 \pm 1.0$ & $1.0 \pm 1.2$ & 0.2169 \\
\hline Barthel index at examination & $98.0 \pm 8.2$ & $98.2 \pm 5.6$ & $99.6 \pm 1.9$ & $93.3 \pm 20.0$ & $96.5 \pm 9.4$ & 0.4729 \\
\hline \multicolumn{7}{|l|}{ eGFR } \\
\hline $5 \mathrm{y}$ prior to examination & $58.9 \pm 17.1$ & $61.7 \pm 16.0$ & $61.6 \pm 17.6$ & $56.5 \pm 20.2$ & $42.9 \pm 6.5$ & 0.0062 \\
\hline 3 y prior to examination & $58.5 \pm 17.5$ & $62.7 \pm 18.8$ & $56.9 \pm 15.9$ & $60.2 \pm 18.5$ & $45.4 \pm 9.2$ & 0.0458 \\
\hline At examination & $58.3 \pm 17.3$ & $60.8 \pm 16.5$ & $59.7 \pm 18.6$ & $57.3 \pm 19.8$ & $46.1 \pm 8.9$ & 0.0438 \\
\hline \multicolumn{7}{|l|}{ Fazekas Deep WMH grade } \\
\hline 5 y prior to examination & $1.0 \pm 1.0$ & $0.8 \pm 0.9$ & $1.0 \pm 0.8$ & $1.2 \pm 1.1$ & $1.8 \pm 1.3$ & 0.0391 \\
\hline 3 y prior to examination & $1.1 \pm 0.9$ & $0.8 \pm 0.8$ & $1.0 \pm 0.8$ & $1.2 \pm 1.1$ & $1.8 \pm 1.2$ & 0.0413 \\
\hline At examination & $1.2 \pm 1.0$ & $0.9 \pm 0.9$ & $1.1 \pm 0.9$ & $1.3 \pm 1.2$ & $2.3 \pm 1.1$ & 0.0039 \\
\hline \multicolumn{7}{|l|}{ Fazekas PVH grade } \\
\hline 5 y prior to examination & $0.8 \pm 0.9$ & $0.8 \pm 0.9$ & $0.6 \pm 0.7$ & $1.2 \pm 1.2$ & $1.1 \pm 1.5$ & 0.0978 \\
\hline 3 y prior to examination & $1.0 \pm 1.0$ & $0.9 \pm 0.9$ & $0.8 \pm 0.9$ & $1.3 \pm 1.2$ & $1.2 \pm 1.4$ & 0.2272 \\
\hline At examination & $1.1 \pm 1.0$ & $1.0 \pm 0.9$ & $0.9 \pm 0.9$ & $1.3 \pm 1.2$ & $1.4 \pm 1.5$ & 0.1359 \\
\hline
\end{tabular}

Table 1. Baseline demographic characteristics. CA, carotid artery; mRS, modified Rankin scale; eGFR, estimated glomerular filtration rate; DWMH, deep white matter hyperintensity; PVH, periventricular hyperintensity.

of severely impaired cognitive domains on Cognistat or total Cognistat score were seen between patients with and without brain infarction $>3 \mathrm{~cm}$ in maximum diameter $(\mathrm{p}=0.6937$ or $\mathrm{p}=0.2385$, respectively).

Risk factors and cognitive impairment. Table 1 shows baseline demographic characteristics by the number of severely impaired domains on Cognistat. At least one domain was severely impaired in 47 patients, $\geq 2$ domains in 19 patients, and $\geq 3$ domains in 10 patients. The eGFR was significantly low and deep WMH grade was significantly high in patients with severe impairment in $\geq 3$ domains compared to other patients. This difference was significant for eGFR at 5 years prior to examination compared to eGFR at 3 years prior to or at examination. Conversely, the difference between deep WMH grade at examination and deep WMH grade at 5 years or 3 years prior to examination was significant.

Association between eGFR or deep WMH and cognitive impairment. Table 2 shows the association between eGFR or deep WMH grade and the number of severely impaired cognitive domains. Univariate analyses revealed that eGFR and deep WMH grade associated with severe impairment in $\geq 3$ domains or $\geq 2$ domains, but not with severe impairment in $\geq 1$ domain. These associations were significant for severe impairment in $\geq 3$ domains compared to in $\geq 2$ domains. The association was significant for eGFR at 5 years prior to examination compared to eGFR at 3 years prior to or at examination. Conversely, this association was significant for deep WMH grade at examination compared to 3 or 5 years prior to examination. No associations between the number 


\begin{tabular}{|c|c|c|c|c|c|c|c|c|c|}
\hline & \multicolumn{9}{|c|}{ Number of severely impaired domains on Cognistat } \\
\hline & \multicolumn{3}{|l|}{$\geq 1$} & \multicolumn{3}{|l|}{$\geq 2$} & \multicolumn{3}{|l|}{$\geq 3$} \\
\hline & OR & $95 \% \mathrm{CI}$ & $\mathbf{P}$ & OR & 95\% CI & $\mathbf{P}$ & OR & $95 \% \mathrm{CI}$ & $\mathbf{P}$ \\
\hline \multicolumn{10}{|c|}{ eGFR (per $1-\mathrm{mL} / \mathrm{min} / 1.73 \mathrm{~m}^{2}$ increase) } \\
\hline $5 y$ prior to examination & 0.98 & $0.96-1.01$ & 0.2140 & 0.95 & $0.91-0.99$ & 0.0045 & 0.89 & $0.82-0.97$ & 0.0004 \\
\hline 3 y prior to examination & 0.98 & $0.95-1.00$ & 0.0540 & 0.97 & $0.94-1.01$ & 0.1020 & 0.94 & $0.88-0.99$ & 0.0084 \\
\hline At examination & 0.99 & $0.96-1.01$ & 0.2431 & 0.97 & $0.94-1.00$ & 0.0419 & 0.95 & $0.90-0.99$ & 0.0116 \\
\hline \multicolumn{10}{|c|}{ Fazekas deep WMH grade (per 1-grade increase) } \\
\hline $5 \mathrm{y}$ prior to examination & 1.57 & $0.94-2.61$ & 0.0755 & 1.89 & $1.09-3.29$ & 0.0211 & 2.32 & $1.14-4.71$ & 0.0172 \\
\hline 3 y prior to examination & 1.59 & $0.96-2.64$ & 0.0611 & 1.99 & $1.15-3.47$ & 0.0124 & 2.47 & $1.23-4.94$ & 0.0090 \\
\hline At examination & 1.71 & $1.06-2.75$ & 0.0198 & 2.24 & $1.33-3.78$ & 0.0015 & 3.32 & $1.61-6.85$ & 0.0003 \\
\hline
\end{tabular}

Table 2. Association between eGFR or deep white matter hyperintensity and the number of severely impaired cognitive domains on Cognistat. eGFR, estimated glomerular filtration rate; DWMH, deep white matter hyperintensity; PVH, periventricular hyperintensity; OR, odds ratio; $\mathrm{CI}$, confidence interval; $\mathrm{WMH}$, white matter hyperintensity.

of severely impaired cognitive domains and eGFR or deep WMH grade were seen in the reference group (data not shown).

Receiver operating characteristic analysis revealed that a cutoff eGFR of $46.8 \mathrm{~mL} / \mathrm{min} / 1.73 \mathrm{~m}^{2}$ at 5 years prior to examination offered the most reliable predictor of severe impairment in $\geq 3$ domains (sensitivity $88.9 \%$, specificity $76.5 \%$, area under the curve (AUC) 0.848 ), while a cutoff of $50.4 \mathrm{~mL} / \mathrm{min} / 1.73 \mathrm{~m}^{2}$ at 5 years prior to examination was the most reliable predictor of severe impairment in $\geq 2$ domains (sensitivity $70.6 \%$, specificity $73.3 \%$, AUC 0.741).

Association between Cognistat scores and eGFR or deep WMH grade. Figure 2 shows linear associations between the absolute value of eGFR and total Cognistat score. The eGFR at 5 years prior to examination was significantly associated with total Cognistat score $\left(\mathrm{r}^{2}=0.11035, \mathrm{p}=0.0032\right)$ compared to eGFR at 3 years prior to examination $\left(r^{2}=0.06455, p=0.0230\right)$ or eGFR at examination $\left(r^{2}=0.0210, p=0.0210\right)$. No association between eGFR and total Cognistat score was seen in the reference group (data not shown). Table 3 shows the correlation between scores for sub-components on Cognistat and eGFR. Orientation, comprehension, repetition, construction, memory, and similarity correlated significantly with eGFR at 5 years prior to examination compared to eGFR at 3 years prior to examination or eGFR at examination.

Figure 3 shows the difference in total Cognistat scores between deep WMH grades. Kruskal-Wallis testing revealed that total Cognistat score differed significantly between deep WMH grades at examination compared to deep WMH grades at 3 or 5 years prior to examination. On the other hand, total Cognistat score did not differ significantly between PVH grades $(\mathrm{p}=0.816$ for $\mathrm{PVH}$ grade at examination, $\mathrm{p}=0.0640$ for $\mathrm{PVH}$ grade at 3 years prior to examination, and $\mathrm{p}=0.1570$ for $\mathrm{PVH}$ grade at 5 years prior to examination). Table 4 shows Cognistat scores by deep WMH grade at examination. Total score, orientation, comprehension, naming, construction, calculation, similarity, and judgement were significantly lower with grade 2 or 3 compared to grade 0 or 1 .

We also tested the association between deep WMH grade and eGFR. Linear regression analysis revealed that deep WMH grade at examination was not associated with eGFR ( $\mathrm{p}=0.1873$ for eGFR at 5 years prior to examination, $\mathrm{p}=0.3674$ for eGFR at 3 years prior to examination, or $\mathrm{p}=0.2705$ for eGFR at examination, respectively).

\section{Discussion}

We found that eGFR was associated with cognitive function in patients who had undergone CEA. This association was significant for eGFR at 5 years prior to cognitive examination compared to eGFR at 3 years prior to or at examination, suggesting that the effects of low eGFR might require a certain period of time to affect cognitive function, and that eGFR might be useful to predict cognitive declines in subsequent years. We also found that deep WMH was associated with cognitive declines. This association was significant for deep WMH at examination compared to deep WMH at 5 years prior to examination, suggesting that deep WMH might influence present cognitive function.

CKD has a well-established association with cognitive impairment. Impaired kidney function in the form of $\mathrm{eGFR}<60 \mathrm{~mL} / \mathrm{min} / 1.73 \mathrm{~m}^{2}$ was associated with a more rapid rate of cognitive decline after adjusting for age, sex, and education in 886 elderly individuals without dementia ${ }^{13}$. This association persisted after excluding participants with $\mathrm{eGFR}<30 \mathrm{~mL} / \mathrm{min} / 1.73 \mathrm{~m}^{2}$, indicating that the association is not limited to severe kidney dysfunction. On the other hand, cognitive decline related to kidney dysfunction was reported to improve after renal transplantation $^{14}$, suggesting that the underlying mechanism of cognitive decline caused by kidney dysfunction is at least partially due to reversible changes rather than irreversible white matter damages. The basis for the relationship between kidney dysfunction and cognitive decline is uncertain, but the shared environmental risk factor hypothesis and the unidirectional causal hypothesis have been proposed as possible mechanisms ${ }^{9}$. CKD creates a toxic vascular and metabolic milieu with substances such as homocysteine, uremic toxins, creatinine, and cystatin C that promote systemic inflammation, oxidative stress, uremia, and systemic vascular endothelial dysfunction ${ }^{7}$. Impaired kidney function was associated with increased risk of intraplaque hemorrhage (odds ratio 1.15 per 20-point decrease in eGFR) in patients undergoing CEA, suggesting a pathway connecting CKD and WMH in patients undergoing $\mathrm{CEA}^{15}$. 

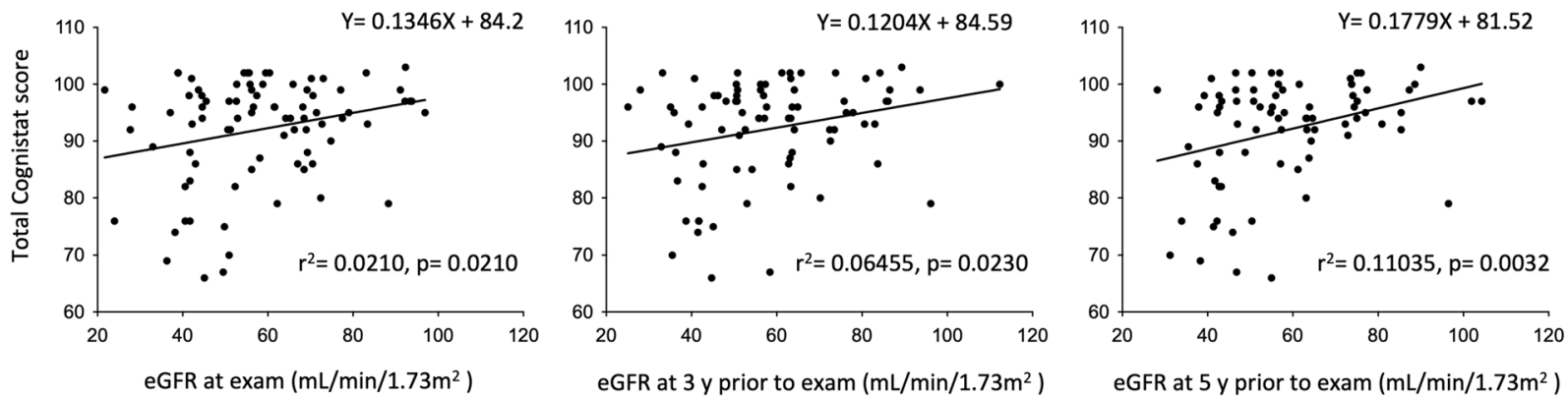

Figure 2. Linear association between absolute value of eGFR and total Cognistat score. The eGFR at 5 years prior to examination shows a stronger association with total Cognistat score than eGFR either at 3 years prior to examination or at examination.

\begin{tabular}{|l|l|l|l|}
\hline & $\begin{array}{l}\text { eGFR at } \\
\text { exam }\end{array}$ & $\begin{array}{l}\text { eGFR 3y prior } \\
\text { to exam }\end{array}$ & $\begin{array}{l}\text { eGFR 5y prior } \\
\text { to exam }\end{array}$ \\
\hline Orientation & 0.0964 & 0.1024 & $0.2640^{*}$ \\
\hline Attention & 0.1291 & 0.1259 & -0.0170 \\
\hline Comprehension & $0.3108^{* *}$ & $0.2595^{*}$ & $0.3644^{* *}$ \\
\hline Repetition & $0.2972 * *$ & $0.2202^{*}$ & $0.3677^{*} *$ \\
\hline Naming & -0.0081 & 0.0187 & -0.0544 \\
\hline Construction & 0.1948 & $0.2460^{*}$ & $0.2242^{*}$ \\
\hline Memory & 0.0656 & 0.1464 & $0.3087^{*} *$ \\
\hline Calculation & 0.1035 & 0.0024 & 0.2158 \\
\hline Similarity & 0.1030 & 0.1813 & $0.2789^{*}$ \\
\hline Judgement & 0.0803 & 0.1676 & 0.0866 \\
\hline
\end{tabular}

Table 3. Spearman's correlation coefficient $\left(r_{s}\right)$ for eGFR and sub-component scores of Cognistat. eGFR, estimated glomerular filtration rate $* \mathrm{p}<0.05 ; * \mathrm{p}<0.01$.

WMH has been shown to be strongly associated with an increased risk of cognitive impairment ${ }^{16}$. Community-based epidemiologic studies have revealed that WMH and silent brain infarct are associated with cognitive decline ${ }^{17-20}$. Another study revealed WMH as one of the differences between cognitive responders and non-responders at 1 year after $\mathrm{CEA}^{4}$. CEA prevented the expected increase in WMH at 1 year after CEA in 14 patients with preoperative cognitive decline ${ }^{1}$, but long-term effects of the persistent WMH after CEA on cognition remain unknown. New ischemic lesions after carotid stenting have been shown to be associated with an increased risk of recurrent cerebrovascular events, but not after $\mathrm{CEA}^{21}$. WMH reduces white matter integrity, resulting in decreased coordinating interactions between different brain regions ${ }^{7}$. Demyelination, loss of oligodendrocytes, and axonal damage are evident in WMH, suggesting the association of chronic hypoperfusion, venous collagenosis, and damage to the blood-brain barrier ${ }^{22}$. These pathological changes might be associated with toxins created by CKD. The Northern Manhattan Study demonstrated that an eGFR of $15-60 \mathrm{~mL} / \mathrm{min} / 1.73 \mathrm{~m}^{2}$ was associated with increased log-WMH volume after adjusting for age, sex, race, and education in 615 subjects ${ }^{23}$. This association was not evident for eGFR $>60 \mathrm{~mL} / \mathrm{min} / 1.73 \mathrm{~m}^{2}$, indicating that eGFR was associated with WMH in moderate to severe CKD, but not in mild CKD. This might provide an explanation as to why WMH was not associated with eGFR in our study, where overall mean eGFR was $58 \mathrm{~mL} / \mathrm{min} / 1.73 \mathrm{~m}^{2}$.

Several studies have reported early changes in cognitive domains mainly at 1-6 months after CEA. Attention, memory, executive function, visuospatial orientation, psychomotor speed, and fluency have been reported to improve shortly after $\mathrm{CEA}^{1,2,24-27}$, while language, working memory, and global cognition deteriorated ${ }^{2,4,28-30}$. Only one study investigated the P300 evoked potential as a marker of cognitive function at 5 years after CEA in 25 patients, but detailed results for specific cognitive functions were not reported ${ }^{31}$. We showed that specific cognitive domains such as orientation, comprehension, repetition, construction, memory, and similarity correlated significantly with eGFR at 5 years prior to examination, suggesting that eGFR might also be useful to predict functional declines in these cognitive domains after several years.

A key strength of this study was that cognitive functions were assessed long after CEA, and that eGFR was measured and MRI was performed at both 5 years and 3 years before examination, but some limitations also need to be considered when interpreting the results. First, this was a retrospective cross-sectional study, not a prospective longitudinal study. We did not perform Cognistat at 5 or 3 years before examination, because Cognistat was only introduced at our institution in 2016. This makes direct evaluation of the impact of eGFR on cognitive changes difficult, but short-term longitudinal studies might be influenced by practice effects. To clarify the impact of eGFR on cognitive changes after CEA, a longitudinal study with a sufficient interval is necessary. Second, this study did not include a control group, only a reference group. Associations between eGFR or deep WMH and 

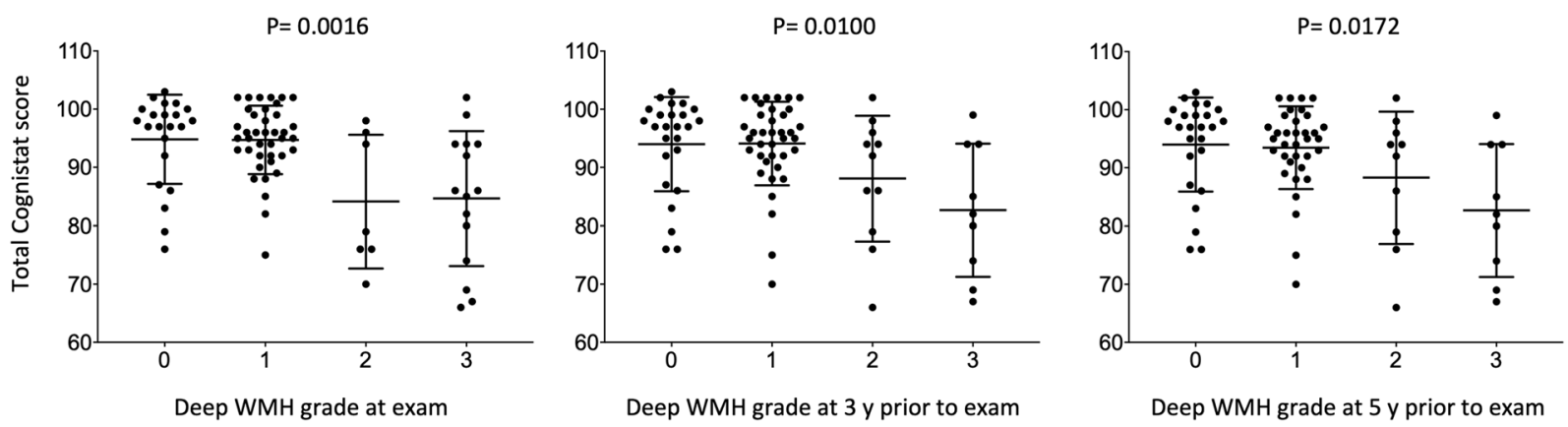

Figure 3. Difference in total Cognistat score between deep white matter hyperintensity (WMH) grade. Total Cognistat scores differ significantly between deep WMH grades at examination compared to deep WMH grades at 3 years or 5 years prior to examination

\begin{tabular}{|l|c|c|l|l|l|l|}
\hline & \multirow{7}{*}{ Overall } & \multicolumn{6}{|l|}{ Fazekas grade for deep white matter hyperintensity } & \multirow{2}{*}{} \\
\cline { 3 - 6 } & $\mathbf{0}$ & $\mathbf{1}$ & $\mathbf{2}$ & $\mathbf{3}$ & P value \\
\hline Total score & $92.0 \pm 9.2$ & $94.8 \pm 7.7$ & $94.7 \pm 5.9$ & $84.1 \pm 11.5$ & $84.7 \pm 11.6$ & 0.0016 \\
\hline Orientation & $9.5 \pm 1.2$ & $9.9 \pm 0.4$ & $9.6 \pm 1.0$ & $9.1 \pm 1.9$ & $8.7 \pm 1.8$ & 0.0227 \\
\hline Attention & $8.2 \pm 3.0$ & $8.6 \pm 2.8$ & $8.8 \pm 2.6$ & $6.6 \pm 3.7$ & $6.7 \pm 3.6$ & 0.0614 \\
\hline Comprehension & $9.2 \pm 1.8$ & $9.7 \pm 1.0$ & $9.5 \pm 1.4$ & $8.3 \pm 2.4$ & $8.0 \pm 2.7$ & 0.0310 \\
\hline Repetition & $9.7 \pm 1.9$ & $9.8 \pm 2.0$ & $9.9 \pm 1.7$ & $8.6 \pm 2.3$ & $9.5 \pm 2.3$ & 0.4406 \\
\hline Naming & $9.6 \pm 0.9$ & $9.6 \pm 0.9$ & $9.9 \pm 0.3$ & $9.0 \pm 1.0$ & $9.3 \pm 1.4$ & 0.0085 \\
\hline Construction & $9.0 \pm 1.8$ & $9.6 \pm 1.4$ & $9.4 \pm 1.6$ & $8.0 \pm 1.7$ & $7.4 \pm 2.0$ & 0.0009 \\
\hline Memory & $7.8 \pm 1.7$ & $8.0 \pm 1.6$ & $7.9 \pm 1.7$ & $8.1 \pm 1.8$ & $7.2 \pm 1.7$ & 0.4436 \\
\hline Calculation & $9.4 \pm 1.4$ & $9.6 \pm 1.3$ & $9.7 \pm 1.0$ & $8.6 \pm 1.9$ & $8.8 \pm 2.0$ & 0.0383 \\
\hline Similarity & $9.6 \pm 1.1$ & $9.7 \pm 1.0$ & $9.8 \pm 1.0$ & $8.4 \pm 1.5$ & $9.3 \pm 1.0$ & 0.0218 \\
\hline Judgement & $10.2 \pm 1.0$ & $10.2 \pm 1.0$ & $10.4 \pm 0.9$ & $9.4 \pm 1.0$ & $9.8 \pm 1.1$ & 0.0477 \\
\hline
\end{tabular}

Table 4. Cognistat scores by deep white matter grade at examination.

cognitive functions were not seen in the reference group, but this does not directly mean that these findings are specific to patients who have undergone CEA. The present findings might be more generalizable observations. Nevertheless, eGFR may offer a useful marker to predict future cognitive decline in patients who have undergone CEA.

\section{Conclusions}

While eGFR correlated with future cognitive decline, deep WMH was associated with present cognitive function in patients who had undergone CEA. Further study is necessary to elucidate whether this association is specific after CEA or represents a generalizable phenomenon.

\section{Data availability}

The datasets generated and/or analyzed during the current study are available from the corresponding author on reasonable request.

Received: 14 June 2019; Accepted: 14 November 2019;

Published online: 28 November 2019

\section{References}

1. Dempsey, R. J. et al. The Preservation of Cognition 1 Year After Carotid Endarterectomy in Patients With Prior Cognitive Decline. Neurosurgery 82, 322-328 (2018).

2. Lal, B. K. et al. Cognitive changes after surgery vs stenting for carotid artery stenosis. J. Vasc. Surg. 54, 691-698 (2011).

3. Lal, B. K. Cognitive function after carotid artery revascularization. Vasc Endovascular Surg 41, 5-13 (2007).

4. Casas-Hernanz, L. et al. Neuropsychological Outcome One Year after Carotid Revascularization: A before-and-after Study. Vasc Specialist Int 33, 146-155 (2017).

5. Carta, M. G. et al. Patients with carotid atherosclerosis who underwent or did not undergo carotid endarterectomy: outcome on mood, cognition and quality of life. BMC Psychiatry 15, 277 (2015).

6. Pearson, S., Maddern, G. \& Fitridge, R. Cognitive performance in patients after carotid endarterectomy. J. Vasc. Surg. 38, 1248-1252 (2003).

7. Bronas, U. G., Puzantian, H. \& Hannan, M. Cognitive Impairment in Chronic Kidney Disease: Vascular Milieu and the Potential Therapeutic Role of Exercise. Biomed Res Int 2017, 2726369 (2017).

8. Toyoda, K. Cerebral small vessel disease and chronic kidney disease. J Stroke 17, 31-37 (2015). 
9. Zammit, A. R., Katz, M. J., Zimmerman, M. E., Bitzer, M. \& Lipton, R. B. Low eGFR is associated with dysexecutive and amnestic mild cognitive impairment. Alzheimers Dement (Amst) 1, 152-159 (2015).

10. Matsuda, O. \& Nakatani, M. The manuals of the japanese version of the neurovehavioral cognitive status examination. (World Plan, 2004).

11. Kiernan, R. J., Mueller, J., Langston, J. W. \& Van Dyke, C. The Neurobehavioral Cognitive Status Examination: a brief but quantitative approach to cognitive assessment. Ann. Intern. Med. 107, 481-485 (1987).

12. Fazekas, F., Chawluk, J. B., Alavi, A., Hurtig, H. I. \& Zimmerman, R. A. MR signal abnormalities at 1.5 T in Alzheimer's dementia and normal aging. AJR Am J Roentgenol 149, 351-356 (1987).

13. Buchman, A. S. et al. Kidney function is associated with the rate of cognitive decline in the elderly. Neurology 73, 920-927 (2009).

14. Radić, J. et al. Kidney transplantation improves cognitive and psychomotor functions in adult hemodialysis patients. Am. J. Nephrol. 34, 399-406 (2011).

15. Wesseling, M. et al. Impaired kidney function is associated with intraplaque hemorrhage in patients undergoing carotid endarterectomy. Atherosclerosis 266, 128-135 (2017).

16. Zhou, G. et al. Cerebral white matter lesions and cognitive function in a non-demented Chinese veteran cohort. J. Int. Med. Res. 36, $115-122$ (2008).

17. Debette, S. et al. Association of MRI markers of vascular brain injury with incident stroke, mild cognitive impairment, dementia, and mortality: the Framingham Offspring Study. Stroke 41, 600-606 (2010).

18. Seshadri, S. et al. Stroke risk profile, brain volume, and cognitive function: the Framingham Offspring Study. Neurology 63, 1591-1599 (2004).

19. van Dijk, E. J. et al. Progression of cerebral small vessel disease in relation to risk factors and cognitive consequences: Rotterdam Scan study. Stroke 39, 2712-2719 (2008).

20. Longstreth, W. T. et al. Incidence, manifestations, and predictors of worsening white matter on serial cranial magnetic resonance imaging in the elderly: the Cardiovascular Health Study. Stroke 36, 56-61 (2005).

21. Gensicke, H. et al. Ischemic brain lesions after carotid artery stenting increase future cerebrovascular risk. J. Am. Coll. Cardiol. 65 , 521-529 (2015).

22. Shi, Y. et al. Cerebral blood flow in small vessel disease: A systematic review and meta-analysis. J. Cereb. Blood Flow Metab. 36, 1653-1667 (2016).

23. Khatri, M. et al. Chronic kidney disease is associated with white matter hyperintensity volume: the Northern Manhattan Study (NOMAS). Stroke 38, 3121-3126 (2007).

24. Usman, R., Jamil, M., Haq, I. U. \& Memon, A. A. Neurocognitive Improvement in Patients Undergoing Carotid Endarterectomy for Atherosclerotic Occlusive Carotid Artery Disease. Ann Vasc Dis 9, 307-311 (2016).

25. Kougias, P. et al. Comparison of domain-specific cognitive function after carotid endarterectomy and stenting. J. Vasc. Surg. 62, 355-361 (2015).

26. Fearn, S. J. et al. Carotid endarterectomy improves cognitive function in patients with exhausted cerebrovascular reserve. Eur J Vasc Endovasc Surg 26, 529-536 (2003).

27. Bossema, E. R., Brand, N., Moll, F. L., Ackerstaff, R. G. A. \& van Doornen, L. J. P. Does carotid endarterectomy improve cognitive functioning? J. Vasc. Surg. 41, 775-781 (2005).

28. Brand, N., Bossema, E. R., Ommen Mv, Mvan, Moll, F. L. \& Ackerstaff, R. G. A. Left or right carotid endarterectomy in patients with atherosclerotic disease: ipsilateral effects on cognition? Brain Cogn 54, 117-123 (2004).

29. Feliziani, F. T. et al. Cognitive performance in elderly patients undergoing carotid endarterectomy or carotid artery stenting: a twelve-month follow-up study. Cerebrovasc. Dis. 30, 244-251 (2010).

30. Ogasawara, K. et al. Postoperative cerebral hyperperfusion associated with impaired cognitive function in patients undergoing carotid endarterectomy. J. Neurosurg. 102, 38-44 (2005).

31. Czerny, M. et al. Sustained cognitive benefit 5 years after carotid endarterectomy. J. Vasc. Surg. 51, 1139-1144 (2010).

\section{Author contributions}

A.N. designed the study, analyzed patient data, and drafted the main manuscript. T.A. and T.K. critically reviewed the manuscript. M.Y. contributed to interpretation of data. Y.O. supervised the study. All authors approved the submitted version of the paper.

\section{Competing interests}

The authors declare no competing interests.

\section{Additional information}

Correspondence and requests for materials should be addressed to A.N.

Reprints and permissions information is available at www.nature.com/reprints.

Publisher's note Springer Nature remains neutral with regard to jurisdictional claims in published maps and institutional affiliations.

(c) (i) Open Access This article is licensed under a Creative Commons Attribution 4.0 International cc) License, which permits use, sharing, adaptation, distribution and reproduction in any medium or format, as long as you give appropriate credit to the original author(s) and the source, provide a link to the Creative Commons license, and indicate if changes were made. The images or other third party material in this article are included in the article's Creative Commons license, unless indicated otherwise in a credit line to the material. If material is not included in the article's Creative Commons license and your intended use is not permitted by statutory regulation or exceeds the permitted use, you will need to obtain permission directly from the copyright holder. To view a copy of this license, visit http://creativecommons.org/licenses/by/4.0/.

(C) The Author(s) 2019 